\title{
Low-frequency Raman scattering in alkali tellurite glasses
}

\author{
ANGELOS G KALAMPOUNIAS \\ Department of Chemical Engineering, University of Patras, P.O. Box 1414, GR 26504, Patras, Greece \\ Also at Foundation for Research and Technology Hellas, Institute of Chemical Engineering and \\ High Temperature Chemical Processes, FORTH/ICE-HT, Patras, Greece
}

MS received 16 January 2008

\begin{abstract}
Raman scattering has been employed to study the alkali-cation size dependence and the polarization characteristics of the low-frequency modes for the glass-forming tellurite mixtures, $0 \cdot 1 \mathrm{M}_{2} \mathrm{O}-0 \cdot 9 \mathrm{TeO}_{2}(\mathrm{M}=\mathrm{Na}$, $\mathrm{K}, \mathrm{Rb}$ and $\mathrm{Cs}$ ). The analysis has shown that the Raman coupling coefficient alters by varying the type of the alkali cation. The addition of alkali modifier in the tellurite network leads to the conversion of the $\mathrm{TeO}_{4}$ units to $\mathrm{TeO}_{3}$ units with a varying number of non-bridging oxygen atoms. Emphasis has also been given to the lowfrequency modes and particular points related to the low-frequency Raman phenomenology are discussed in view of the experimental findings.
\end{abstract}

Keywords. Tellurites; Raman spectroscopy; alkali tellurite glasses.

\section{Introduction}

Tellurite glasses have scientific and technological interest due to their attractive physical properties such as low melting points, high dielectric constant (Stanworth 1952; Ulrich 1964), high refractive index (Stanworth 1952) and good infrared transmissivity (Burger et al 1985). Compared with silicate and phosphate glasses, tellurites exhibit relatively higher refractive indices, dielectric constants and lower phonon energies. The refractive indices allow the utilization of these glasses for non-linear optical materials (Shioya et al 1995) and the phonon energies have atleast two important consequences, the improved infrared transmission up to $6 \mu \mathrm{m}$ and the low multiphonon decay rates compared to other network oxide glasses. Furthermore, tellurite glasses possess chemical durability and are water resistive (Redman and Chen 1967).

Currently, there exists a large amount of literature regarding the structure of pure $\mathrm{TeO}_{2}$ and $x \mathrm{M}_{2} \mathrm{O}-(1-x)$ $\mathrm{TeO}_{2}$ (M: alkali oxide, $x$ : mole fraction) glasses with Raman spectroscopy (see e.g. Sekiya et al 1992; Tatsumisago et al 1994a; Noguera et al 2003). However, a limited number of articles have focused on the low-frequency region of the Raman spectra and the comparison between binary glasses with the same concentration in alkali oxide but with different types of cation.

In the present paper, we report the room temperature Raman spectra of the $0 \cdot 1 \mathrm{M}_{2} \mathrm{O}-0 \cdot 9 \mathrm{TeO}_{2}(\mathrm{M}=\mathrm{Na}, \mathrm{K}, \mathrm{Rb}$ and $\mathrm{Cs}$ ) glasses. The data are discussed in terms of the glass local structure and the effect of the incorporated

(angelos@chemeng.upatras.gr) cation in the glass. Emphasis has been given in the analysis of the low-frequency Raman features and the relation of the observed data to the cation size.

\section{Experimental}

The reagent grade chemicals, tellurium (IV) oxide $(99.99 \%$ purity) and sodium, potassium, rubidium and cesium carbonates (99.95\% purity) were purchased from Alfa Aesar. The appropriate amounts of polycrystalline $\mathrm{TeO}_{2}$ and $\mathrm{M}_{2} \mathrm{CO}_{3}(\mathrm{M}=\mathrm{Na}, \mathrm{K}, \mathrm{Rb}$ and $\mathrm{Cs})$ were mixed and melted in platinum crucibles at $700-800^{\circ} \mathrm{C}$ for $15-30 \mathrm{~min}$. The homogenized bubble-free liquids were quenched using the conventional method by dipping the crucible into cold water. Heating of the samples was performed at temperatures near the glass transition temperature in order to avoid cracking due to relieved internal stresses generated by quenching.

Raman spectra were recorded using the $514.5 \mathrm{~nm}$ line of an $\mathrm{Ar}^{+}$laser as excitation source. The laser power at the sample was set at $\sim 200 \mathrm{~mW}$. The scattered light was resolved by a triple monochromator/spectrograph Jobin Yvon T64000, set at the double subtractive mode configuration for better stray light rejection and for sharp cutoff of the exciting laser line at low frequencies. The spectral resolution was fixed at $2 \mathrm{~cm}^{-1}$, while the scattered light was detected by a CCD detector. A system of achromatic and anti-reflection coated lenses was used to collect the right angle scattered light. Both polarized (VV: vertical polarization of incident laser-vertical analysis of scattered light) and depolarized ( $\mathrm{VH}$ : vertical polarization of incident laser-horizontal analysis of scattered light) 
scattering geometries were employed for glasses. This was achieved with the aid of a set of Glan and GlanThompson polarizers (Hale) with extinction coefficients better than $10^{-6}$ and $10^{-7}$, respectively. A calibration procedure with the aid of a $\mathrm{CCl}_{4}$ sample was often taking place in order to check the polarization and correct for possible drifts of the monochromator's gratings. Polycrystalline solids were measured with the verticalunpolarized (VU) configuration. Accumulation times were adjusted to result in a very high signal-to-noise ratio; few minutes depending on the sample temperature were sufficient for this. All room temperature spectra were recorded in a backscattering geometry under a microscope where the excitation laser beam was focused onto the sample by a microscope objective $(50 \times / 0 \cdot 55)$. Since the spectral conditions are the same for all $0 \cdot 1 \mathrm{M}_{2} \mathrm{O}-0 \cdot 9 \mathrm{TeO}_{2}$ $(\mathrm{M}=\mathrm{Na}, \mathrm{K}, \mathrm{Rb}$ and $\mathrm{Cs}$ ) glasses at room temperature, the Raman intensities could be quantitatively correlated within an error of $\sim 5 \%$.

\section{Results and discussion}

Based on Raman spectroscopic results the structure of pure $\mathrm{TeO}_{2}$ and tellurium oxide based glasses reveals two basic findings: First, the pure $\mathrm{TeO}_{2}$ consists of $\mathrm{TeO}_{4}$ trigonal bipyramids (tbps) in which one equatorial site of the $s p^{3} d$ hybrid orbitals is occupied by a lone pair of electrons and the other two equatorial and axial sites are occupied by oxygen atoms. Second, the addition of alkali and/or alkaline earth modifiers to pure $\mathrm{TeO}_{2}$ network causes a change of the Te coordination polyhedron from $\mathrm{TeO}_{4}$ tbp to $\mathrm{TeO}_{3}$ trigonal pyramid (tp) in which one of the Te $s p^{3}$ hybrid orbitals is occupied by a lone pair of electrons (Sekiya et al

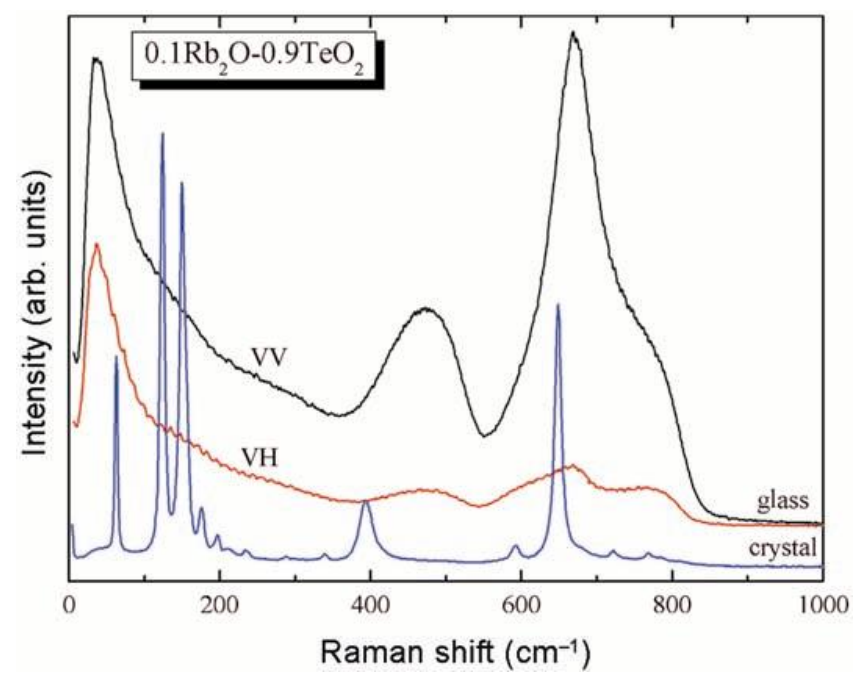

Figure 1. Stokes-side polarized (VV) and depolarized (VH) Raman spectra of $0 \cdot 1 \mathrm{Rb}_{2} \mathrm{O}-0 \cdot 9 \mathrm{TeO}_{2}$ glass at room temperature. The spectrum of the corresponding crystal is also shown for comparison (Spectral conditions: excitation line: $514.5 \mathrm{~nm}$, laser power: $200 \mathrm{~mW}$, resolution: $2 \mathrm{~cm}^{-1}$, CCD detector).
1992; Tatsumisago et al 1994a; Noguera et al 2003). Figure 1 shows the room temperature polarized and depolarized Raman spectra of $0 \cdot 1 \mathrm{Rb}_{2} \mathrm{O}-0 \cdot 9 \mathrm{TeO}_{2}$ glass. The spectrum of the corresponding crystal is also shown for comparison. The spectrum of the glass is characterized by three polarized bands at high frequencies, viz. a broad band near $475 \mathrm{~cm}^{-1}$, an intense band at $\sim 670 \mathrm{~cm}^{-1}$ and a shoulder band near $\sim 770 \mathrm{~cm}^{-1}$. These bands have been attributed to the bending mode of Te-O-Te or O-Te-O linkages, to stretching modes of the $\mathrm{TeO}_{4}$ tbp, to stretching mode of the $\mathrm{TeO}_{3}$ tp units, respectively (Sekiya et al 1992; Tatsumisago et al 1994b; Noguera et al 2003). At lower frequencies the spectrum is dominated by a well resolved asymmetric peak, viz. the Boson peak, and several lowlying vibrational lines centred at $\sim 130$ and $\sim 280 \mathrm{~cm}^{-1}$. These bands observed in the low-frequency region are assigned to a bending vibration of $\mathrm{TeO}_{3}$ tp having two or three NBO atoms (Sekiya et al 1992; Tatsumisago et al $1994 \mathrm{~b})$. The Raman bands of $0 \cdot 1 \mathrm{Rb}_{2} \mathrm{O}-0 \cdot 9 \mathrm{TeO}_{2}$ crystal are similar to that observed in paratellurite $a-\mathrm{TeO}_{2}$ crystal

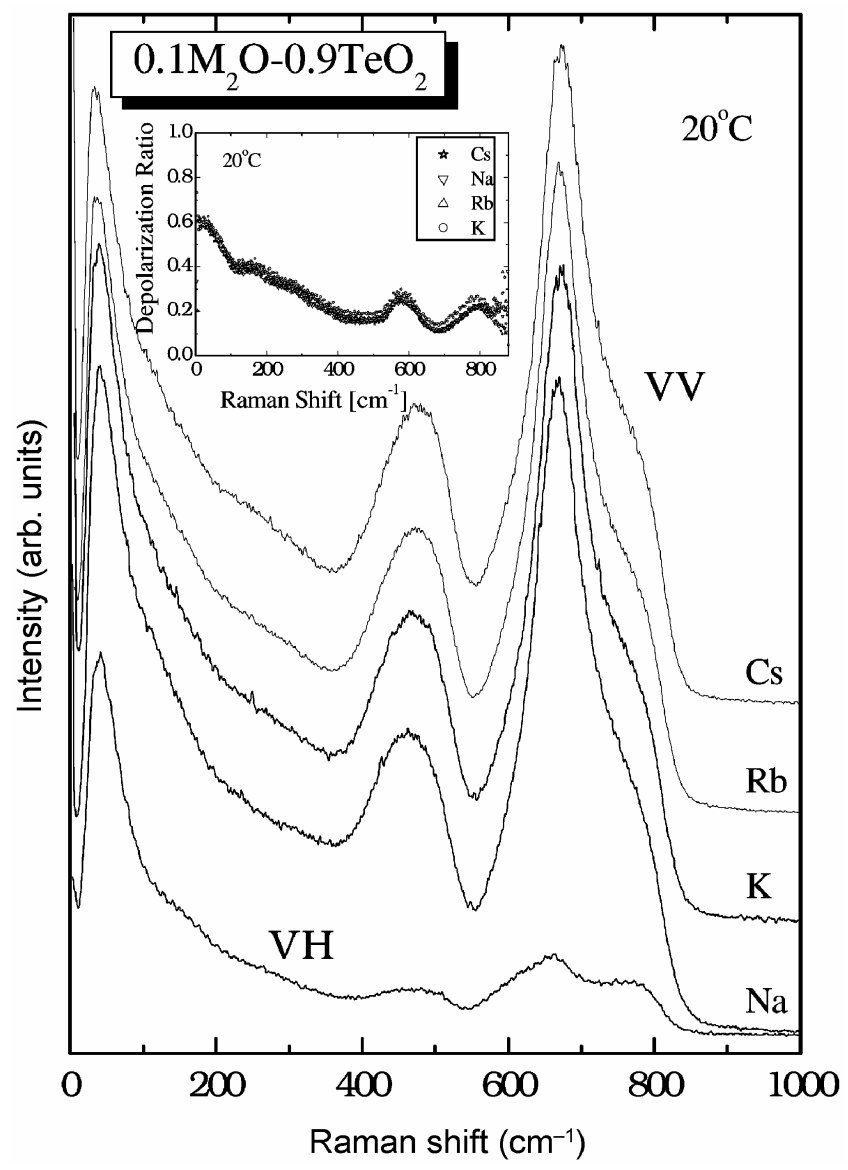

Figure 2. Stokes-side VV Raman spectra of $0 \cdot 1 \mathrm{M}_{2} \mathrm{O}-0 \cdot 9 \mathrm{TeO}_{2}$ $(\mathrm{M}=\mathrm{Na}, \mathrm{K}, \mathrm{Rb}$ and $\mathrm{Cs})$ glasses at room temperature. The depolarized spectrum is shown only for $\mathrm{Na}$ containing glass, inset: depolarization ratio for all glasses at room temperature for comparison (Spectral conditions: excitation line: $514.5 \mathrm{~nm}$, laser power: $400 \mathrm{~mW}$, resolution: $2 \mathrm{~cm}^{-1}$, CCD detector). 
(Pine and Dresselhaus 1972). In the paratellurite lattice, the lone pairs of Te-atoms are always oriented vis-á-vis and the two neighbouring $\mathrm{TeO}_{4}$ tbps, also known as disphenoids, are interconnected via a corner forming a threedimensional network structure. A comparison of the Raman spectra of the glass and of the corresponding crystal reveals that their structure is not similar.

Figure 2 shows the room temperature polarized Raman spectra of $0 \cdot 1 \mathrm{M}_{2} \mathrm{O}-0 \cdot 9 \mathrm{TeO}_{2}$ glasses where $\mathrm{M}=\mathrm{Na}, \mathrm{K}, \mathrm{Rb}$ and Cs. The depolarized spectra were measured for all systems but only $\mathrm{Na}$ containing glass depolarized spectrum is shown here. The inset of figure 2 illustrates the depolarization ratios for all glasses at room temperature. It seems that the polarization characteristics are similar for all glasses containing different types of alkali.

The low-frequency region (below $150 \mathrm{~cm}^{-1}$ ) of the Raman spectra of alkali tellurite glasses consists of two contributions. Firstly, the Boson peak, an asymmetric intense peak, which is absent from the spectra of the cor-

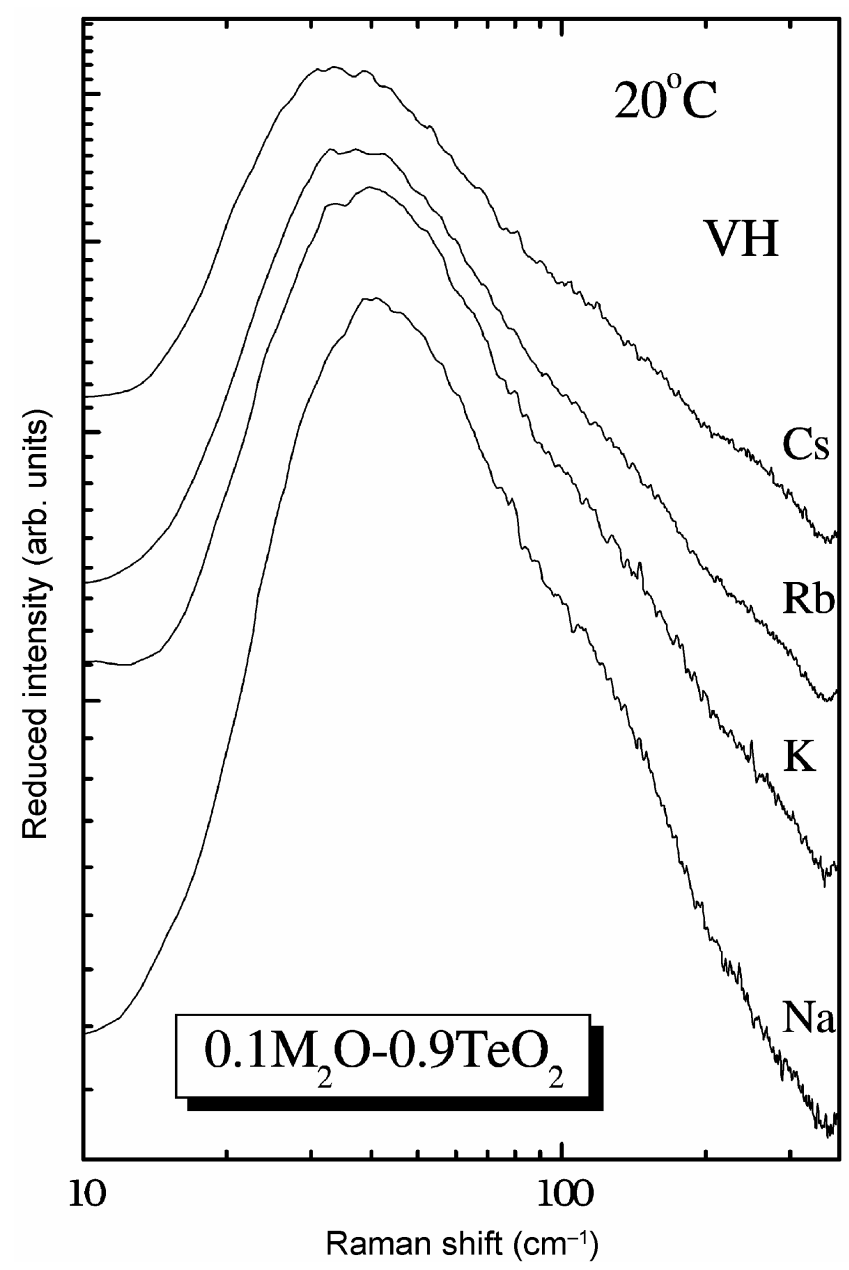

Figure 3. Stokes-side, low-frequency reduced Raman spectra of $0 \cdot 1 \mathrm{M}_{2} \mathrm{O}-0 \cdot 9 \mathrm{TeO}_{2}(\mathrm{M}=\mathrm{Na}, \mathrm{K}, \mathrm{Rb}$ and $\mathrm{Cs})$ glasses for $\mathrm{VH}$ scattering geometry at room temperature in a $\log -\log$ representation. responding crystals. The nature and origin of this spectral characteristic of amorphous materials have received considerable attention. Secondly, there is the quasielastic (QE) line that appears in the spectrum as a zero centred line with a half width at half height of about $10-15 \mathrm{~cm}^{-1}$. It is usually assigned to a fast relaxation process with characteristic time in the sub-picosecond time scale. In an effort to elucidate the microscopic origin of the Boson peak, its spectral features such as peak frequency, polarization characteristics and intensity have to be studied in detail as a function of some external parameter. In the present study, this is attempted by varying the type of modification i.e. the kind of alkali modifier. The lowfrequency Raman spectra, for the series of glasses studied
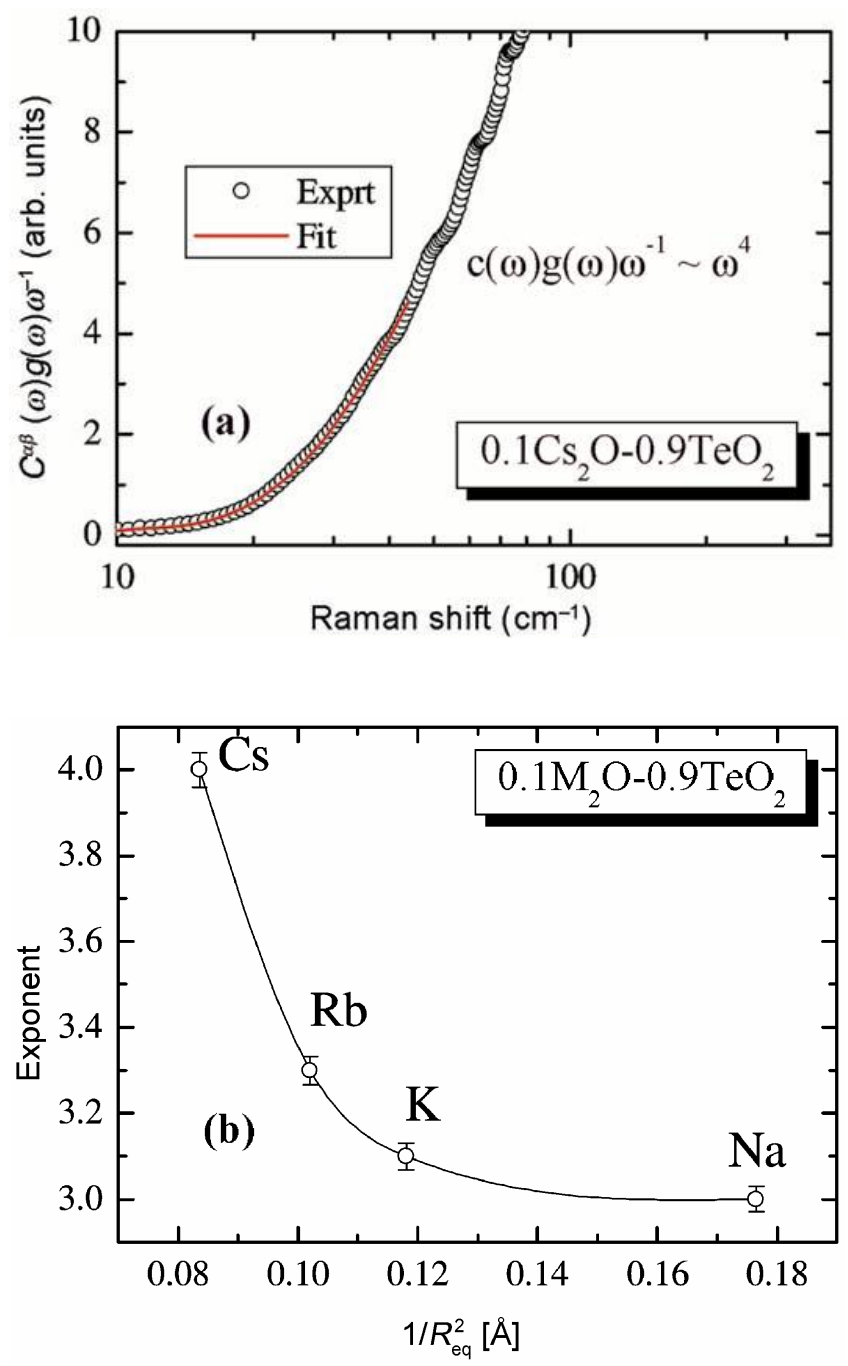

Figure 4. (a) Representative semi-logarithmic plot of the reduced depolarized Raman spectra (open circles) for $0 \cdot 1 \mathrm{Cs}_{2} \mathrm{O}-$ $0 \cdot 9 \mathrm{TeO}_{2}$ glass. The solid line represents power law fit to the data as described in the text and (b) exponent of power law vs $1 / \sqrt{R_{\text {eq }}^{2}}$ of alkali cations for $0 \cdot 1 \mathrm{M}_{2} \mathrm{O}-0 \cdot 9 \mathrm{TeO}_{2}(\mathrm{M}=\mathrm{Na}, \mathrm{K}, \mathrm{Rb}$ and $\mathrm{Cs}$ ) glasses at room temperature. Solid line is drawn as guide to the eye. 
here are shown in figure 3 . The spectra are presented in the reduced representation, i.e.

$$
I^{\mathrm{red}}(\omega)=I^{\operatorname{expt}}(\omega) / \omega[n(\omega, T)+1]=g(\omega) C^{\alpha \beta}(\omega) / \omega^{2},
$$

where $g(\omega)$ is the vibrational density of states (VDoS), $C^{\alpha \beta}(\omega)$ the Raman coupling coefficient and $n(\omega, T)$ the Bose-Einstein occupation number. The different components labeled, $\alpha \beta$, denote particular polarization geometries (VV and $\mathrm{VH}$ ).

The spectrum is generally believed to arise from a breakdown in the Raman selection rule in the disordered material allowing essentially all vibrational modes to participate in the scattering process. This fact led to the conclusion that the Raman scattering is first-order scattering and is closely related to the density of states. In order to isolate the function, $g(\omega)$, the expression

$$
I_{\text {red }}^{\alpha \beta}(\omega)=\frac{I^{\alpha \beta}(\omega)}{\omega^{-1}[n(\omega, T)+1]}=C^{\alpha \beta}(\omega) g(\omega),
$$

is frequently used. The frequency dependence of the coupling coefficient cannot be determined through a direct comparison of Raman and neutron scattering data without taking account for the polarization dependence of $C^{\alpha \beta}(\omega)$. In many studies the Raman coupling coefficient was determined by considering only the depolarized reduced Raman intensity. Detailed analysis of the depolarization ratio for several oxides and chlorides in a wide temperature range showed strong frequency dependence (Papatheodorou and Yannopoulas 2002). $C^{\alpha \beta}(\omega)$ is a very specific property of the studied system related to the way that vibrational motion modulates the polarizability of the medium and as such differs among various liquids. Figure $4(a)$ shows the frequency dependence of the $C^{\alpha \beta}(\omega) g(\omega) \omega^{-1}$ factor in the low-frequency region $\left(<100 \mathrm{~cm}^{-1}\right)$ for the

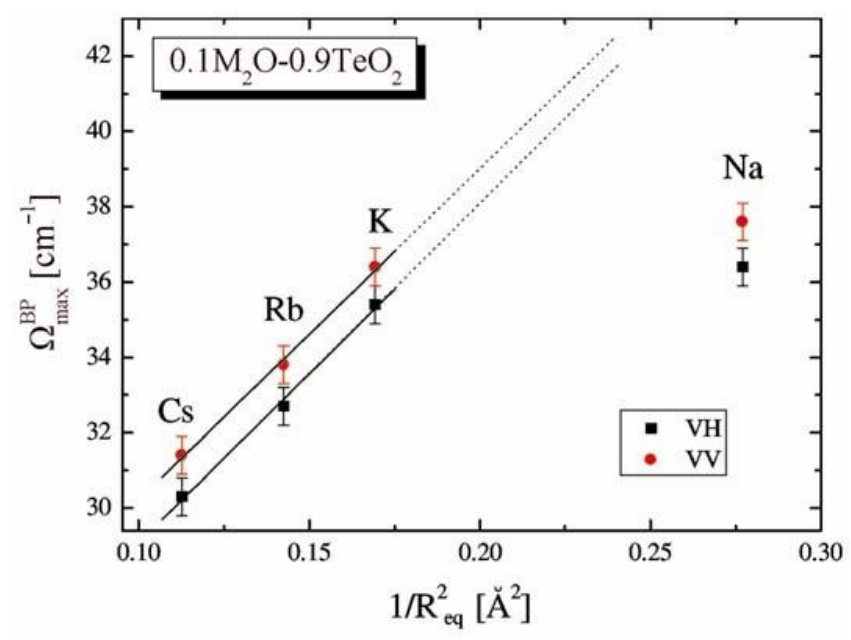

Figure 5. Frequency of the Boson peak maximum vs $1 / \sqrt{R_{\mathrm{eq}}^{2}}$ of alkali cations for $0 \cdot 1 \mathrm{M}_{2} \mathrm{O}-0 \cdot 9 \mathrm{TeO}_{2}(\mathrm{M}=\mathrm{Na}, \mathrm{K}, \mathrm{Rb}$ and $\mathrm{Cs})$ glasses at room temperature and $\mathrm{VV}, \mathrm{VH}$ scattering geometries. Solid lines are drawn as guide to the eye. room temperature $0 \cdot 1 \mathrm{Cs}_{2} \mathrm{O}-0 \cdot 9 \mathrm{TeO}_{2}$ glass. Similar results have been obtained for all $0 \cdot 1 \mathrm{M}_{2} \mathrm{O}-0 \cdot 9 \mathrm{TeO}_{2}$ glasses. The factor, $C^{\alpha \beta}(\omega) g(\omega)$, has been multiplied by $\omega^{-1}$ in order not to suppress the low frequencies. This figure reveals the following facts. First, the limiting low-frequency spectra do not show remnants of the QE component suggesting that it is rather a symmetric line. Second, the frequency dependence of the Raman coupling coefficient can be elucidated. Indeed, the equation

$$
C^{\alpha \beta}(\omega) g(\omega) \omega^{-1} \propto \omega^{x},
$$

is valid as shown in figure 4(a).

Using this power law proportionality in the above equation we obtained the overall exponent, $x$. The results are shown in figure 4 (b) for all $0 \cdot 1 \mathrm{M}_{2} \mathrm{O}-0 \cdot 9 \mathrm{TeO}_{2}$ glasses at room temperature. It is noteworthy that the resultant values for the power law exponent vary from 4 to 3 , depending on the cation. Accordingly, the exponent for the net $C^{\alpha \beta}(\omega) g(\omega)$ product assumes mean values close to 5, $4 \cdot 3,4 \cdot 1$ and 4 for $\mathrm{Cs}, \mathrm{Rb}, \mathrm{K}$ and $\mathrm{Na}$, respectively. In the context of the rigid conception at such very low frequencies the vibrational density of states follows fairly well the Debye law, i.e. $g(\omega) \propto \omega^{2}$. Then, the low frequency behaviour of the Raman coupling coefficient follows a power law with exponents close to $3,2 \cdot 3,2 \cdot 1$ and 2 for $\mathrm{Cs}, \mathrm{Rb}, \mathrm{K}$ and $\mathrm{Na}$, respectively.

In many oxide glasses, as also in the case of tellurite glasses, the presence of low-lying vibrational modes obstructs the quantitative analysis of the BP. In order to overcome this problem we have performed a simple deconvolution procedure on the low-frequency Raman spectra using the fewest possible adjustable parameters. The BP is described with a log-normal distribution, the simplest asymmetric distribution and the low-lying vibrations as Gaussian distributions.

The alkali modifier dependence of the BP is evident in figure 3. As regards the frequency of the BP there is a clear dependence on the square root of the alkali mass as figure 5 reveals. The larger the size of the modifier, the lower the frequency of the BP. This is reasonable considering that modifying cations participate in the vibrating 'structural unit' in the glass that gives rise to the BP. The creation of a larger number of $\mathrm{TeO}_{3}$ tps in the sequence $\mathrm{Na} \rightarrow \mathrm{Cs}$ is also in agreement with the cation size dependence of the BP frequency. Similar behaviour exhibits the Boson peak frequency in the alkali metaphosphate glasses where also exist some dependence of the cation on the low-frequency scattering (Miller 1979). Further, the depolarization ratio of the various glasses is frequency dependent over the spectral range of the BP exhibiting a clear systematic decrease (see inset in figure 2).

\section{Conclusions}

In this paper we present the Raman spectra of $0 \cdot 1 \mathrm{M}_{2} \mathrm{O}-$ $0 \cdot 9 \mathrm{TeO}_{2}(\mathrm{M}=\mathrm{Na}, \mathrm{K}, \mathrm{Rb}$ and $\mathrm{Cs})$ alkali tellurite glasses in 
an effort to elucidate the changes caused in the low frequency behaviour of the Raman coupling coefficient by altering the type of the alkali cation. The network structure of the tellurite glass is composed of $\mathrm{TeO}_{4}$ trigonal bipyramid and $\mathrm{TeO}_{3}$ trigonal pyramid units. On adding alkali modifier in the network, the $\mathrm{TeO}_{4}$ units are converted to $\mathrm{TeO}_{3}$ units with a varying number of nonbridging oxygen atoms. The low-frequency Raman spectral features reveal cation dependence as in the case of alkali metaphosphate glasses. The dependence of the maximum of the Boson peak as a function of cation size has also been determined and the results reveal a monotonic decrease.

\section{Acknowledgements}

The author wishes to thank Professor G N Papatheodorou for providing experimental facilities and Dr S N Yannopoulos for helpful discussions.

\section{References}

Burger H, Vogel W and Kozhukharov V 1985 Infrared Phys. 25395 Miller Ph J 1979 J. Chem. Phys. 71997

Noguera O, Merle-Mejean T, Mirgorodsky A P, Smirov M B, Thomas P and Champarnaud-Mesjard J-C 2003 J. Non-Cryst. Solids 33050

Papatheodorou G N and Yannopoulos S N 2002 Molten salts: From fundamentals to applications (ed.) M Gaune-Escard (Dordrecht: Kluwer Academic) pp 47-106

Pine A S and Dresselhaus G 1972 Phys. Rev. B5 4087

Redman M J and Chen J H 1967 J. Am. Ceram. Soc. 50523

Sekiya T, Mochida N, Ohtsuka A and Tonokawa M $1992 \mathrm{~J}$. Non-Cryst. Solids 144128

Shioya K, Kmatsu T, Kim H G, Sato R and Matsusita K 1995 J. Non-Cryst. Solids 18916

Stanworth J E 1952 Nature 169581

Tatsumisago M, Minami T, Kowada Y and Adashi H 1994a Phys. Chem. Glasses 3589

Tatsumisago M, Lee S-K, Minami T and Kowada Y 1994b J. Non-Cryst. Solids 177154

Ulrich D R 1964 J. Am. Ceram. Soc. 47595 